NASZA DERMATOLOGIA Online

OUR DERMATOLOGY Online

\section{HLA-DPDQDR IS EXPRESSED IN LESIONAL SKIN FROM PATIENTS WITH AUTOIMMUNE SKIN DISEASES}

\author{
Ana Maria Abreu Velez ${ }^{1,3}$, Juliana Calle-Isaza ${ }^{2}$, \\ Michael S. Howard ${ }^{1}$ \\ ${ }^{1}$ Georgia Dermatopathology Associates, Atlanta, Georgia, USA \\ ${ }^{2}$ Clinic El Poblado, Medellin, Colombia, South America \\ ${ }^{3}$ Department of Dermatology, School of Medicine, University of Antioquia, Medellin, \\ Colombia, South America
}

Source of Support: Georgia Dermatopathology Associates, Atlanta, Georgia, USA (MSH, AMAV); Mineros SA, Medellin, Colombia, SA; Embassy of Japan in Colombia, Medellin; University of Antioquia, Medellin Competing Interests: None
Corresponding author: Prof. Ana Maria Abreu Velez, M.D., Ph.D. abreuvelez@yahoo.com

\begin{abstract}
Introduction: Human genes responsible for human antigen presentation and transplant rejection functions are located on the short arm of Chromosome 6, and are called the Major Histocompatibility Complex (MHC). Moreover, the primary physiologic function of MHC molecules is to present peptides to $\mathrm{T}$ lymphocytes. MHC molecules are integral components of the ligands that most $\mathrm{T}$ cells recognize, since the $\mathrm{T}$ cell receptor (TCR) has specificity for complexes of foreign antigenic peptides, as well as self-MHC molecules.

Aim: Our investigation attempts to investigate the presence of HLA-DPDQDR within lesional skin biopsies from patients affected by autoimmune skin blistering diseases (ABDs).

Materials and Methods: We utilized immunohistochemistry (IHC) to evaluate the presence of HLA-DPDQDR in lesional skin biopsies of patients affected by ABDs. We tested 30 patients with endemic pemphigus foliaceus (EPF), 15 controls from the EPF endemic area, and 15 biopsies from healthy controls from the USA. We also tested archival biopsies from patients with selected ABDs, including 30 patients with bullous pemphigoid (BP), 20 with pemphigus vulgaris (PV), 8 with pemphigus foliaceus (PF), 14 with dermatitis herpetiformis (DH) and 2 with epidermolysis bullosa acquisita (EBA).

Results: Most ABD biopsies stained positive for HLA-DPDQDR in the lesional blisters and/or inflamed neurovascular neuroplexus areas in the superficial dermis, and also at mesenchymal-endothelial like-cell junctions in the dermis. In BP, EBA and EPF, the HLA-DPDQDR staining was also seen in dermal eccrine sweat gland coils and ducts.

Conclusion: We document that HLA-DPDQDR is expressed in several anatomic areas of lesional skin in patients with ABDs. Notably, HLADPDQDR positivity was also consistently present in areas of the classic immune response, including epidermal intercellular junctions in pemphigus, and at basement membrane sites in bullous pemphigoid and other subepidermal blistering diseases.
\end{abstract}

Key words: HLA-DPDQDR; autoimmune skin diseases; endemic pemphigus foliaceus

Abbreviations: Bullous pemphigoid (BP), pemphigus vulgaris (PV), pemphigus foliaceus (PF), dermatitis herpetiformis (DH), epidermolysis bullosa acquisita (EBA), immunohistochemistry (IHC), direct and indirect immunofluorescence (DIF and IIF), hematoxylin and eosin (H\&E), basement membrane zone (BMZ), intercellular staining between keratinocytes (ICS), pemphigus vulgaris (PV), autoimmune blistering skin diseases (ABDs), endemic pemphigus foliaceus in El-Bagre, Colombia (El Bagre-EPF), Human Leukocyte Antigen (HLA), Major Histocompatibility Complex (MHC), T cell receptor (TCR).

\section{Introduction}

The Major Histocompatibility Complex (MHC) is central to the adaptive immune response [1]. One of the most polymorphic genetic systems, the Human Leukocyte Antigen (HLA) system is divided into Class I (HLA-A, B and C) and Class II (HLA-DP, DQ and DR) molecules [1-3]. HLA-DP, DQ, and DR represent three differing isotypes, each being highly polymorphic [1-3]. Different HLA-DP variants can be protective, or alternatively, risk factors for infectious diseases, immune dysfunction, and autoimmunity [1-3]. Few studies have investigated the presence of HLA Class II in lesional skin from patients affected by ABDs [4-6]. 
Our present investigation attempted to study the presence of HLA-DP, DQ, and DR in multiple ABDs by performing immunohistochemistry (IHC) stains on lesional skin biopsies.

\section{Material and Methods \\ Subjects of study}

We tested 30 biopsies from patients affected by EPF in El Bagre, Colombia, South America (El Bagre-EPF) and 15 normal controls from the endemic area [3-5]. We also utilized 15 control skin biopsies from plastic surgery reduction patients in the USA, taken from the chest and/or abdomen. We also studied 15 biopsies from healthy controls from the USA. We tested archival biopsies from patients with selected ABDs, including 30 patients with bullous pemphigoid (BP), 20 with pemphigus vulgaris (PV), 8 with pemphigus foliaceus (PF), 14 with dermatitis herpetiformis (DH) and 2 with epidermolysis bullosa acquisita (EBA). Biopsies were fixed in 10\% buffered formalin, then embedded in paraffin and cut at 4 micron thicknesses. The tissue was then submitted for hematoxylin and eosin (H\&E) and IHC staining, performed as previously described [7-11]. In addition, we also tested biopsies from the archival files of two private, board certified dermatopathology laboratories in the USA; these patients underwent primary diagnostic biopsies, and were not taking immunosuppressive therapeutic medications at the time of biopsy. We evaluated 20 biopsies from bullous pemphigoid (BP) patients, 20 from patients with pemphigus vulgaris $(\mathrm{PV}), 8$ patient biopsies with pemphigus foliaceus $(\mathrm{PF})$, 12 from patients with dermatitis herpetiformis (DH) and 3 from epidermolysis bullosa acquisita. For all of the El Bagre area patients and controls, we obtained written consent, as well as Institutional Review Board permission from the local hospital. The archival biopsies were IRB exempt due to the lack of patient identifiers. In both dermatopathology laboratories, each biopsy also was sent for direct immunofluorescence performed as previous described [7-11], for correlation with the $\mathrm{H} \& \mathrm{E}$ diagnoses.

\section{IHC staining}

The staining of the antibodies was evaluated utilizing hematoxylin-counterstained histologic sections. IHC staining was performed as previously described [7-11]. For IHC, we utilized a Dako monoclonal mouse anti-human HLA-DP, DQ, and DR Clone CR3/43 antibody.

\section{Statistical analysis}

For statistical analysis, the non-parametric Mann-Whitney U-test was used to calculate significant levels for all measurements. Values of $p<0.05$ were considered statistically significant.

\section{Results}

Among patients with El Bagre EPF, 23/30 exhibited positive staining in the upper dermal blood vessels and perivascular inflammatory infiltrates with a significance of $p<0.05$. Also, positivity was seen around the neurovascular supply structures of sebaceous glands, and around dermal blood vessels surrounding eccrine ducts (Fig. 1 - 3). Only two controls from the endemic area displayed positive staining, specifically in some upper dermal perivascular infiltrates $(p<0.05)$; controls from the USA stained uniformly negative $(p<0.05)$. Among BP patients, 18/20 stained positive for HLA-DP, DQ and DR in neurovascular packages around dermal eccrine gland ducts. Positivity was also noted under subepidermal blisters, along the bases of the blisters and within dermal endothelial/mesenchymal cell junction-like structures, especially in the middle dermis $(p<0.05)$. In PV patients, 14/20 stained positive within upper dermal perivascular infiltrates, above intraepidermal blisters and around dermal eccrine gland ducts $(p<0.05)$ (Figs. 1 - 3). In patient biopsies with PF, 6/8 stained positive within the epidermal stratum granulosum, and around neurovascular packages around eccrine ducts. Among patients with $\mathrm{DH}$, 9/12 exhibited positive staining, primarily under subepidermal blisters, as well as in superficial dermal perivascular infiltrates $(p<0.05)$. The 3 cases of EBA stained positive around blood vessels in the middle and upper dermis, with some staining in eccrine sweat glands and in the areas of inflammation below the blisters (Fig. 1 - 3). Figure 1 documents the most common patterns of HLA-DP, DQ, and DR staining positivity found in the patients with ABDs. Please also notice focal cells with positive staining in the epidermis of most of the ABDs.
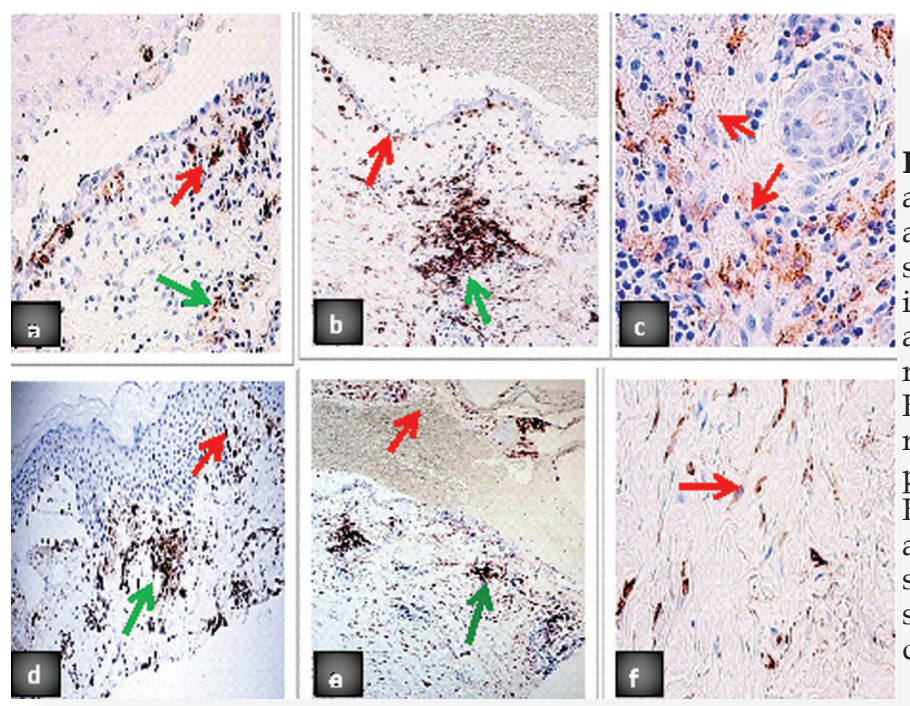

Figure 1. $\mathbf{a}$ and $\mathbf{b}$. HLA-DP, DQ and DR positive staining, under an intraepidermal blister in a case of PV (brown staining; red arrows), as well as around upper dermal blood vessels (brown staining; green arrows). c. HLA-DP, DQ and DR positive staining in a BP case, accentuated around dermal neurovascular bundles around an eccrine gland duct (brown staining; red arrows). d. A representative case of $\mathrm{DH}$, demonstrating positive staining to HLA-DP, DQ, and DR in sub epidermal blisters (brown staining; red arrow) as well as around an upper dermal neurovascular plexus (brown staining; green arrow). e. A case of PV, positive for HLA-DPDQDR above an intradermal blister (brown staining; red arrow), as well as around upper dermal blood vessels (brown staining; green arrow). f. A PV case, with positive HLA-DPDQDR staining on cell junction-like structures between cells of the dermal extracellular matrix (brown staining; red arrow). 

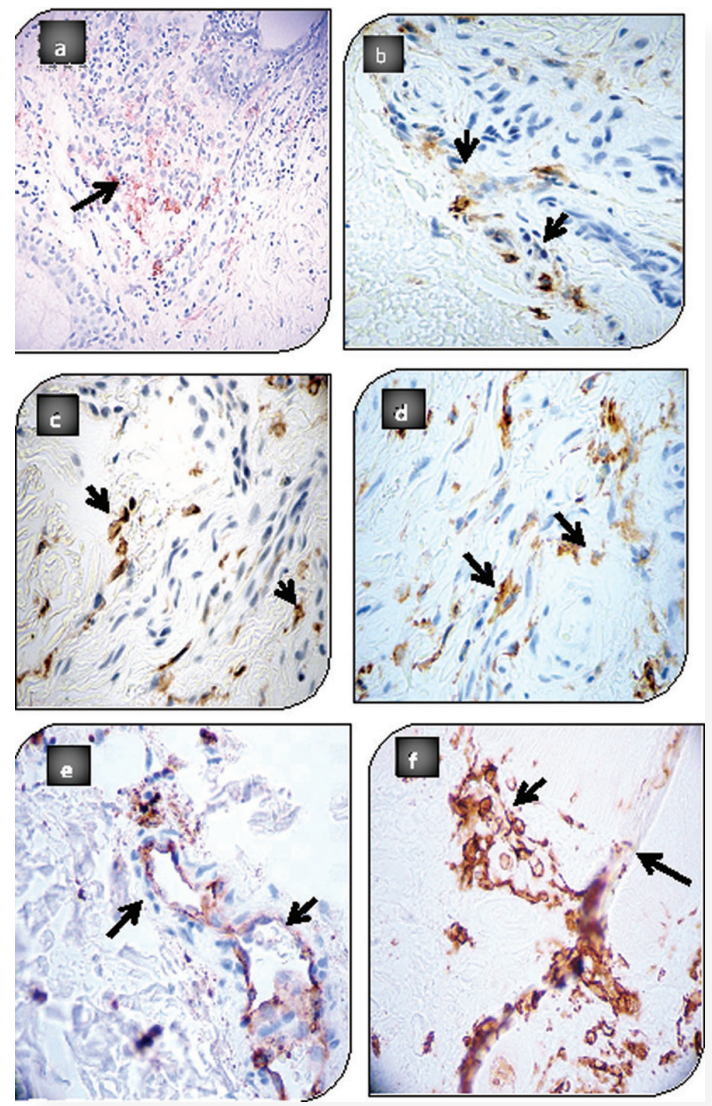

Figure 2. a. A DH case, with positive HLA-DP, DQ, and DR staining around the upper neurovascular plexus subjacent to the blister(brown staining; black arrow). b and c, A BP case demonstrating positive staining in $b$ at the deep dermal neurovascular plexus, and in c around eccrine ducts (brown staining; black arrows). $\mathbf{d}$ and $\mathbf{e}, \mathrm{A}$ case of EBA, with positive HLA-DP, DQ, and DR staining in the upper dermal neurovascular plexus of the skin in $\mathrm{d}$, and in e around dermal blood vessels and eccrine glands (brown staining; black arrows). f A DH case with positive HLA-DP, DQ, and DR staining in the upper dermal neurovascular plexus and eccrine ductus (brown staining; black arrows). In this case we didn't use hematoxylin counterstaining.
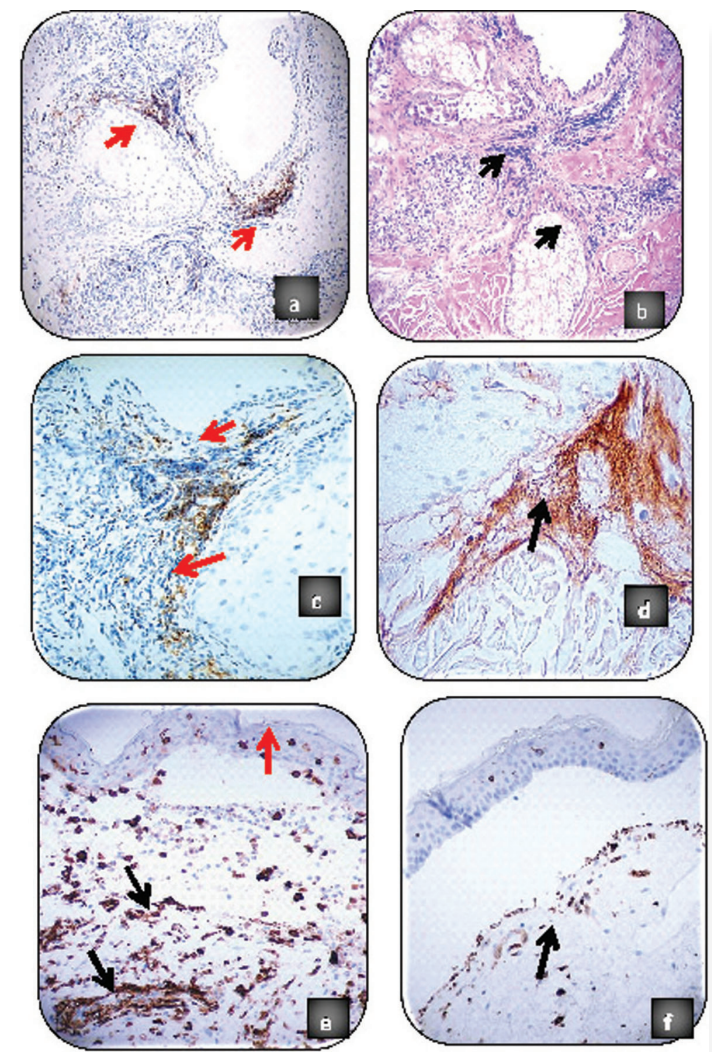

Figure 3. a. A PV case, demonstrating positive staining with HLA-DP, DQ, and DR around dermal neurovascular packages below the blister (brown staining; red arrows) (200X). b. Same case as in a; H\&E staining of the section confirms a correlating lymphohistiocytic infiltrate, present among neurovascular packages that feed the sebaceous glands (black arrows). c. Same case as in $a-b$, with positive HLA-DP, DQ, and DR staining around dermal neurovascular packages supplying pilosebaceous units (brown staining; red arrows) (100X). d. The same case as in a-c, demonstrating positive staining with anti-Complement/C3c against dermal neurovascular packages (brown staining; black arrow) (400X). e. An EBA case, with positive staining on epidermal cells above the blister (brown staining; red arrow) and also around dermal blood vessels (brown staining; black arrows). f. A BP case, with generally linear staining under the blister along the basement membrane zone (brown staining; brown arrow), and around dermal blood vessels. Please also note positive staining on some cells in the epidermis.

\section{Discussion}

Several previous studies, often utilizing serum, have addressed the HLA haplotype distribution utilizing ABD cases and controls [5,6,12-15]. These studies concluded that some HLA alleles strongly predispose toward and/or protect against ABDs.

Antibodies against HLA-DPDQDR principally label B lymphocytes, lymph node interdigitating reticulum cells, Langerhans cells and non-Langerhans macrophages. The specific antibody we utilized is known to react with the alpha and beta-chains of all products of the DP, DQ and DR subregions. Our study is one of the few demonstrating the presence of cells carrying appropriate molecules to react in situ with this antibody to multiple HLA Class II antigens. Our study also demonstrates that antigen recognition, and possibly also the active immune response, does not only occur solely in blister areas in pemphigus and/or pemphigoids, as traditionally thought.

These processes also likely occur in other areas where we have previously shown ABD immune reactivity and cell signaling, specifically areas staining positive for ribosomal protein S6ps240, cyclo-oxygenase 2, proteases and protease inhibitors. These areas specifically include neurovascular supplies to multiple skin appendageal structures, eccrine gland coils and ducts, and mesenchymal/endothelial cell junction-like structures in the dermis [16-21]. 


\section{Conclussion}

The full significance of our findings is unknown. Future research is needed to confirm and expand our research findings, and to address specific reasons for HLA-DP, DQ, and DR positivity in cases of ABDs.

\section{REFERENCES}

1. Saha I, Mazzocco G, Plewczynski D. Consensus classification of human leukocyte antigen class II proteins. Immunogenetics. 2013;65:97-105.

2. Greenbaum J, Sidney J, Chung J, Brander C, Peters B, Sette A. Functional classification of class II human leukocyte antigen (HLA) molecules reveals seven different supertypes and a surprising degree of repertoire sharing across supertypes. Immunogenetics. 2011;63:325-35

3. van Lith M, McEwen-Smith RM, Benham AM. HLA-DP, HLADQ, and HLA-DR have different requirements for invariant chain and HLA-DM. J Biol Chem. 2010;285:40800-8.

4. Tunca M, Musabak U, Sagkan RI, Koc E, Akar A. Association of human leukocyte antigen class II alleles with pemphigus vulgaris in a Turkish population. J Dermatol. 2010;37:246-50.

5. Zakka LR, Reche P, Ahmed AR. Role of MHC Class II genes in the pathogenesis of pemphigoid. Autoimmun Rev. 2011;11:40-7.

6. Abreu Velez AM, Robles EV, Howard MS. A new variant of endemic pemphigus foliaceus in El-Bagre, Colombia: the HardyWeinberg-Castle law and linked short tandem repeats. N Am J Med Sci. 2009;1:169-78.

7. Abreu Velez AM, Howard MS, Smoller BR. Atopic dermatitis with possible polysensitization and monkey esophagus reactivity. N Am J Med Sci. 2010;2:336-40.

8. Abreu Velez AM, Howard MS, Yi H, Gao W, Hashimoto T, Grossniklaus HE. Neural system antigens are recognized by autoantibodies from patients affected by a new variant of endemic pemphigus foliaceus in Colombia. J Clin Immunol. 2011;31:356-68. 9. Abreu Velez AM, Howard MS, Hashimoto T, Grossniklaus HE. Human eyelid meibomian glands and tarsal muscle are recognized by autoantibodies from patients affected by a new variant of endemic pemphigus foliaceus in El-Bagre, Colombia, South America. J Am Acad Dermatol. 2010;62:437-47.

10. Abreu Velez AM, Howard MS, Hashimoto T. Palms with a polyclonal autoimmune response in patients affected by a new variant of endemic pemphigus foliaceus in Colombia, South America. Eur J Dermatol. 2010;20:74-81.
11. Abreu Velez AM, Smith JG Jr, Howard MS. Neutrophil extracellular traps (NETS), IgD, myeloperoxidase (MPO) and antineutrophil cytoplasmic antibody (ANCA) associated vasculitides. North Am J Med Sci. 2009;1:309-13.

12. Piovezan BZ, Petzl-Erler ML. Both qualitative and quantitative genetic variation of MHC class II molecules may influence susceptibility to autoimmune diseases: the case of endemic pemphigus foliaceus. Hum Immunol. 2013;74:1134-40.

13. Párnická Z, Švecová D, Javor J, Shawkatová I, Buc M. High susceptibility to pemphigus vulgaris due to HLA-DRB $1 * 14: 54$ in the Slovak population. Int J Immunogenet. 2013;40:471-5.

14. Koc CK, Sallakci N, Akman-Karakaş A, Alpsoy E, Yegin O. Human leukocyte antigens class I and class II in patients with pemphigus in southern Turkey. Int J Dermatol. 2013;52:53-8.

15. Esmaili N, Mortazavi H, Chams-Davatchi C, Daneshpazhooh M, Damavandi MR, Aryanian Z, et al. Association between HLADQB1*03:01 and Bullous pemphigoid in Iranian patients. Iran J Immunol. 2013;1:1-9.

16. Abreu Velez, AM, Googe PB, Howard MS. Ribosomal protein S6pS240 is expressed in lesional skin from patients with autoimmune skin diseases. North Am J Med Sci. 2013;5:604-8.

17. Abreu Velez AM, Oliver J, Howard MS. Immunohistochemistry studies in a case of dermatitis herpetiformis demonstrate complex patterns of reactivity. Our Dermatol Online. 2013;4(Suppl.3):627-30. 18. Abreu Velez AM, Googe PB, Howard MS. In situ immune response in skin biopsies from patients affected by autoimmune blistering diseases. Our Dermatol Online. 2013;4(Suppl.3):606-12.

19. Abreu Velez AM, Roselino AM, Howard MS. Mast cells, Mast/ Stem Cell Growth Factor receptor (c-kit/CD117) and IgE may be integral to the pathogenesis of endemic pemphigus foliaceus. Our Dermatol Online. 2013;4(Suppl.3):596-600.

20. Abreu Velez AM, Calle J, Howard MS. Cyclo-oxygenase 2 is present in the majority of lesional skin from patients with autoimmune blistering diseases. Our Dermatol Online. 2013;4:476-8.

21. Abreu Velez AM, Yepes-Naranjo MM, Avila IC, Londoño ML, Googe PB, Velásquez-Velez JE, et al. Tissue inhibitor of metalloproteinase 1, Matrix metalloproteinase 9, alpha-1 antitrypsin, metallothionein and urokinase type plasminogen activator receptor in skin biopsies from patients affected by autoimmune blistering diseases. Our Dermatol Online. 2013;4:275-80. 